Y.-H. Chu, N.B. Suntzeff, J.E. Hesser, and D.A. Bohlender, eds.

\title{
Birth of Supernova Remnant 1987A
}

\author{
George Sonneborn, C. S. J. Pun \\ NASA's Goddard Space Flight Center, Code 681, Greenbelt, MD 20771, \\ $U S A$
Peter Garnavich, Robert Kirshner
Harvard/Smithsonian Center for Astrophysics, Cambridge, MA 02139, $U S A$

\begin{abstract}
Recent observations of SN 1987A made with HST/STIS show that the shock interaction of supernova debris with the innermost edge of the circumstellar ring has begun.
\end{abstract}

\section{Observations and Discussion}

The discovery of circumstellar (CS) gas close to SN 1987A quickly led to the realization that the SN debris would soon collide with the CS gas (Fransson et al. 1989). Increasing radio and X-ray emission from SN 1987A since 1991 indicate that some type of interaction was taking place with gas inside the inner CS ring. Recent observations of SN 1987A with the Space Telescope Imaging Spectrograph (STIS) on HST have measured the velocity and location of the collision between high-velocity ejecta $\left(v \sim 15,000 \mathrm{~km} \mathrm{~s}^{-1}\right)$ and CS gas (Sonneborn et al. 1998). The high-velocity $\mathrm{Ly} \alpha$ and $\mathrm{H} \alpha$ emission is associated with the reverse shock of this collision (Michael et al. 1998).

STIS (Sonneborn et al. 1998) and WFPC2 (Garnavich et al. 1997) data also reveal the presence of a second interaction occuring in a highly localized region. This so-called "hot spot" is associated with the forward shock encountering a region of dense gas on the inner-most edge of the inner ring. The spatial relationship between the two shock signatures are illustrated schematically in Figure 1. The observations indicate that the long-awaited collision of the SN debris and the inner circumstellar ring has begun and should increase in brightness many fold across the electromagnetic spectrum in the coming years.

\section{References}

Fransson, C., et al. 1989, ApJ, 336, 429

Garnavich, P., et al. 1997, IAU Circular No. 6710

Michael, E., et al. 1998, ApJ, 492, L143

Sonneborn, G., et al. 1998, ApJ, 492, L139 


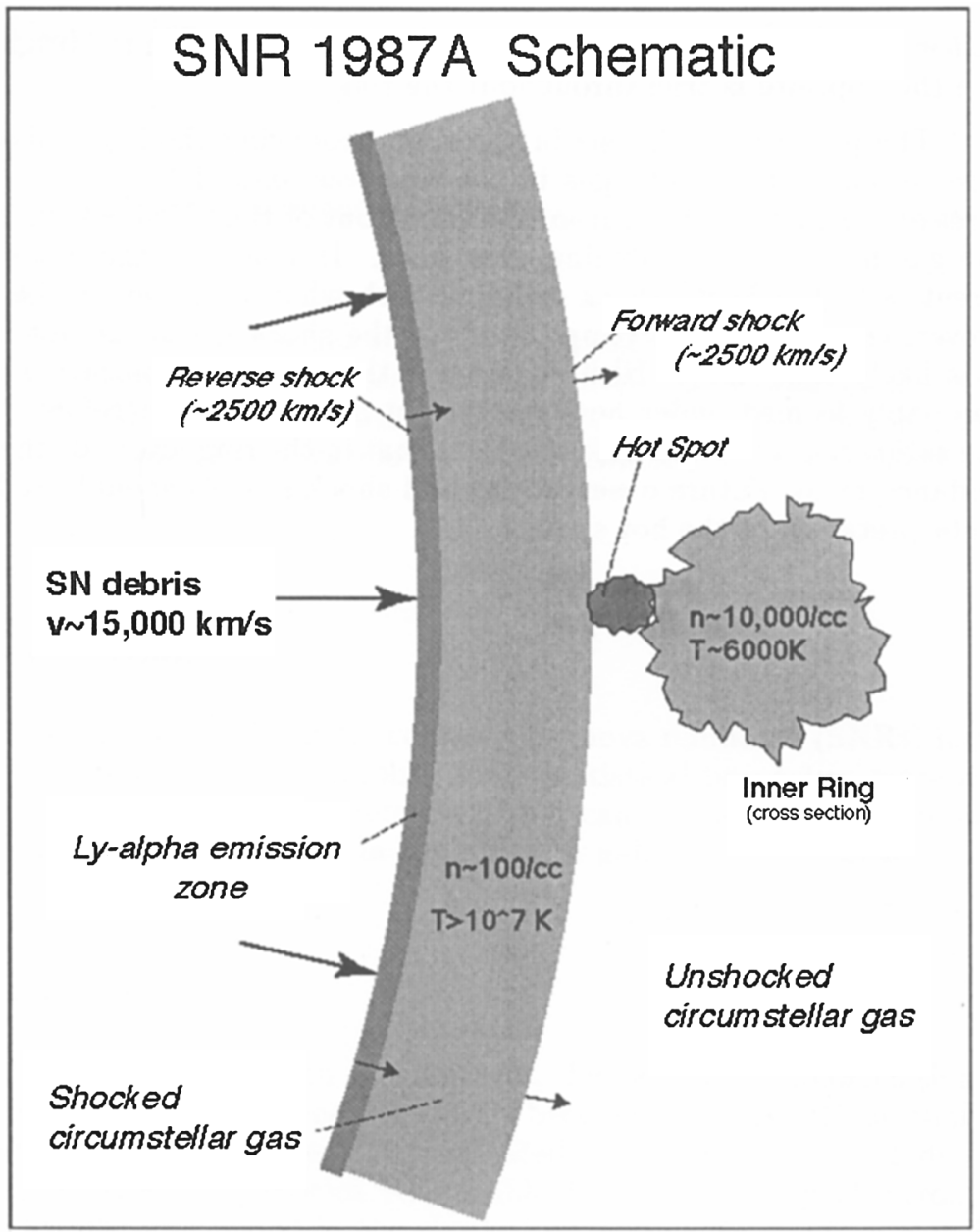

Figure 1. Schematic diagram of the shock interaction around SN $1987 \mathrm{~A}$. The Ly- $\alpha$ emission originates at the reverse shock where lowdensity, high-velocity $\left(\sim 15,000 \mathrm{~km} \mathrm{~s}^{-1}\right)$ neutral debris from the SN explosion collides with circumstellar gas. This CS gas lies interior to the well-known CS ring, has a low density $\left(n_{e} \sim 100 \mathrm{~cm}^{-3}\right)$, and originated as mass lost from the progenitor after the CS ring formed. This collision is driving a shock into the CS gas at $\sim 2500 \mathrm{~km} \mathrm{~s}^{-1}$, raising the temperature in the shocked gas above $10^{7} \mathrm{~K}$, and is the source for the radio and X-ray emission. The "hot spot" is the result of the forward shock from this collision encountering the innermost protrusion of the much denser circumstellar ring $\left(n_{e}>10^{4} \mathrm{~cm}^{-3}\right)$. 


\section{Discussion}

Nolan Walborn: Can you explain why $\mathrm{H} \alpha$ is stronger than [NII] in the brightened knot, while the opposite is true throughout the ring?

Sonneborn: The physical conditions in the ring, producing the line emission is one of pure recombination. The gas in the ring was ionized by the brief (few hours) burst of ionizing radiation from the break out of the SN shock on $23 \mathrm{Feb}$ 1987. The gas has been recombining ever since. In contrast, the hot spot is very different, a high velocity shock colliding with what we believe to be dense gas. However, the density and temperature of the shocked gas are not known today. It is likely that the [N II] lines are not the dominant ionization stages and are probably formed under non-equilibrium conditions. Therefore, the $[\mathrm{N}$ II] $/ \mathrm{H} \alpha$ line ratios could be very different from that in the ring, even for the same $\mathrm{N} / \mathrm{H}$ abundance ratio. Future observations and shock models should produce a physical interpretation of the hot spot. 